\title{
Psychopathology
}

Addiction 121

Adolescents 312

Affective disorder 285

Aggression 223

Alexithymia 170

Alienation 137

Antisaccades 180

Anxiety disorders 389

Arab 345

Asperger's syndrome 8

Assessment 189

Attention bias 79

- deficit hyperactivity disorder, adolescents 69

Attentional bias 25

- maintenance 25

Attitude 262

Audibility 137

Audible thoughts 137

Auditory verbal hallucinations 137

Autism 357

Autistic spectrum disorders 8

\section{Behavior 262}

Behavioural disorder 63

Bipolar disorder 1, 285

Borderline personality disorder 25, 79, 180,369

Brief Psychiatric Rating Scale 345

\section{Capgras 127}

Characteristics of Delusions Rating Scale 373

Childhood trauma 33

Children 209, 312

Classification 389

- of mental disorders 335

Communication 216

Comorbidity 8, 33, 88, 389

Comprehensive diagnosis 252

Context representation 275

Coping strategies 17

Delusion(s) 189, 373

Delusional misidentification syndrome 127

Dementia 300

Depersonalization 141

Depression 41, 121, 159

Depressive disorder 197

Derealization 141
Diagnosis 292

Diagnostic stability 285

Diamorphine 88

Disability 262

Dissociation 33, 104

Dissociative disorders 104

- experiences scale 104

Embodiment 327

Emotional faces 25, 79

Emotions 381

Estrangement 300

Eugène Minkowski 319

Expert rating 373

Explanatory model 230

Explication 319

External validity 369

Face recognition 127

Family environment 312

First-degree relatives 141

Formal thought disorder 275

Fregoli 127

Gedankenlautwerden 137

Gender 159

Genetic syndrome 63

Grade of membership analysis 53

Helmut Rennert 335

Hermeneutics 319

Heroin-assisted treatment 88

Hyperautomaticity 327

Hyperreflectivity 327

ICD-10 110

ICF 262

Immigration 17

Impulsivity 180, 223

Incapacities 262

Inhibition 275

Initial orienting 79

Inner speech 137

Intellectual disability 63

Internet use, excessive 121

Intersubjectivity 268

Interview 189, 319

Latin Americans 17

Longitudinal study 240
Major depression 312

Measurement 150, 189, 373

Medical consultations 216

Methadone maintenance treatment 88

Mood and anxiety disorders 8

Motivation 262

Multidimensional 189

Multidimensionality 373

Narcissism 150

Neurocognitive disorder 300

Neuropsychology 275

Non-clinical population 240

Non-verbal behaviour 216

Obsessive-compulsive disorder 209, 389

- -, children 69

- spectrum disorders 389

Onset 197

Panic disorder 170

Parental psychopathology 209

Participation 262

Patient-reported outcomes 1

Performance 127

Perplexity 357

Personality 159,381

- disorder(s) 110, 159, 197

Phenomenology 41, 268, 327

Phenotype 69

Philosophy 252

Physical abuse 104

Praecox feeling 216, 292

Psychiatric assessment 41

- diagnosis 268

- nosology 335

Psychiatry 252

Psychopathology 17, 63, 262, 319, 345

Psychosis 8, 33, 230

Psychotherapy 170

Questionnaire development 150

Randomized controlled trial 369

Reliability 41, 292

Religion 230

Rubinstein-Taybi 63

Scale 381

Schizoaffective disorder 285 
Schizophrenia 33, 96, 127, 137, 141, 216, 223, 230, 275, 292, 300, 327, 345, 357

- spectrum disorders 141

- subtypes 53

Self-experience 96

Self-report 110

Sexual abuse 104

Short form 150

Single depressive episode 197

Spirituality 230

Stability 240

State emotions 180

Stop signal test 180

Stress 17

Stressful life events 159
Stroop test 180

Structured interview 110

Subjectivity 268

Subsyndromal symptoms 1

Suicide 121, 223

Symptoms, negative 223

-, positive 223

Technical delusion 96

Temperament 240, 381

TEMPS-A 240

Thought insertion 137

Tic disorder 69

Treatment outcome 197
Unitary psychosis 335

Universal genesis 335

Validation 373

Validity 41, 110

Visual dot probe 25

Voices 137

Volition 262

Windelband 252

Working memory 275 